\title{
Prognostic significance of nemo-like kinase in nasopharyngeal carcinoma
}

\author{
SIZE CHEN $^{1,2^{*}}$, ZHIJIAN MA ${ }^{3 *}$, XUEMEI CHEN ${ }^{4}$ and JIREN ZHANG ${ }^{1}$

\begin{abstract}
${ }^{1}$ Department of Oncology, The Affiliated Second Hospital of Southern Medical University, Guangzhou, Guangdong 510282;
${ }^{3}$ Department of Radiation Oncology, Cancer Center of Sun Yat-sen University, Guangzhou, Guangdong 510060;

${ }^{4}$ School of Public Health and Tropic Medicine, South Medical University, Guangzhou, Guangdong 510515, P.R. China
\end{abstract} \\ ${ }^{2}$ Department of Oncology, First Affiliated Hospital of Guangdong Pharmaceutical University, Guangzhou, Guangdong 510080;
}

Received July 27, 2013; Accepted March 6, 2014

DOI: $10.3892 / \mathrm{mmr} .2014 .2190$

\begin{abstract}
Nasopharyngeal carcinoma (NPC) is a highly metastatic malignancy, which is highly prevalent in Southeast Asia and North Africa. Recent studies implicated the critical role of nemo-like kinase (NLK) in tumor biology. However, the functional role of NLK in NPC has yet to be elucidated. In the present study, the significance of NLK positivity in NPC was examined. NLK expression was evaluated by immunohistochemistry in a relatively large sample of patients with NPC $(n=352)$ from December 1, 2002 to December 1, 2009. The correlation between the NLK expression status and clinicopathological features and prognosis was investigated. Univariate and multivariate Cox regression models were developed to evaluate the association between the NLK status and the relative risks for relapse and mortality. In total, $54 \%(190 / 352)$ of NPC samples were identified as positive for NLK. By contrast, all 176 specimens of adjacent normal tissue were negative for NLK. NLK positivity was associated with tumor extent, regional lymph node status and distant metastases. A Kaplan-Meier survival analysis demonstrated that patients with NLK-positive NPC exhibited significantly shorter disease-free survival (DFS) and overall survival (OS). Furthermore, Cox regression analysis revealed that NLK positivity was an unfavorable prognostic indicator of DFS and OS in NPC, independent of other features. Additionally, NLK-positive patients with NPC without distant metastases were more likely to relapse compared with NLK-negative patients with NPC without distant metastases. The present
\end{abstract}

Correspondence to: Dr Jiren Zhang, Department of Oncology, The Affiliated Second Hospital of Southern Medical University, 253 Industrial Avenue, Guangzhou, Guangdong 510282, P.R. China E-mail: nfzhangjiren@126.com

*Contributed equally

Key words: nasopharyngeal carcinoma, nemo-like kinase, prognosis, biomarker study indicates that NLK is a good prognostic marker for NPC.

\section{Introduction}

Nasopharyngeal carcinoma (NPC) is a tumor derived from the epithelial cells that cover the surface of and line the nasopharynx (1). Worldwide, its highest incidence occurs in Southern China, with an age-standardized incidence rate varying from 20 to 50 cases per 100,000 individuals (2). NPC, often accompanied by early lymph node metastasis, is difficult to detect due to its hidden location and no marked clinical manifestations at the early stage (3). To date, the major clinical screening method for NPC is to detect Epstein-Barr virus (EBV) infection in the serum. However, EBV infection is present in a number of other diseases and not all NPC carcinogenesis cases are associated with EBV (4). Furthermore, numerous clinical experiments demonstrated that there is low sensitivity and specificity for diagnosis using EBV-associated methods for NPC screening. Thus, the requirements of a diagnostic marker are not met and the discovery of the molecular biomarkers of NPS are likely to help improve the treatment regimes and predict the prognosis for patients with NPC.

Nemo-like kinase (NLK) is an evolutionarily conserved serine/threonine protein kinase and belongs to the extracellular signal-regulated kinases/microtubule-associated protein kinase family (5). Previous data have shown that NLK is involved in tumor biology. A mitogenic potential of NLK in hepatocellular carcinoma was demonstrated by small interfering (si)RNA-mediated disruption of NLK, which inhibited the proliferation of Hep3B cells and arrested cell cycle transition (6). NLK-silenced Gallbladder carcinoma cell lines demonstrated a decelerated growth rate and alleviated migration ability, indicating the involvement of NLK in the proliferation and migration of gallbladder carcinoma (7). A subsequent investigation has shown that the overexpression of NLK is an independent prognostic factor for patients with gallbladder carcinoma (8).

Although NLK is implicated in the pathogenesis of cancers, its biological functions in NPC have yet to be fully elucidated. In the present study, the correlation between clinicopathological 
features and NLK positivity involving 352 patients with NPC and the prognostic significance of NLK were investigated.

\section{Materials and methods}

Patients and samples. NPC biopsies were collected from 352 patients diagnosed with primary NPC and from 176 specimens of adjacent nasopharyngeal tissue at the Affiliated Second Hospital of Southern Medical University in Guangzhou, China, from December 1, 2002 to December 1, 2009. All the patients had not received any treatment prior to surgery. The cause of mortality was determined according to their medical record.

Formalin-fixed, paraffin-embedded tissues were collected from the Affiliated Second Hospital of Southern Medical University at the time of surgical resections being performed. All the biopsies were histologically confirmed by two pathologists in a blind manner. The clinical stage of NPC was determined according to the tumor, node, and metastasis (TNM) classification system of the American Joint Committee on Cancer/Union for International Cancer Control, and the histological type was designated according to the World Health Organization criteria. Follow-up data included the survival and disease status (disease-free, recurrence or metastasis), along with dates of the events and cause of mortality.

All patients gave informed consent prior to participation in the present study, which was approved by the Ethics Committees of Southern Medical University (Guangzhou, China) and performed in line with the Declaration of Helsinki.

Histological assessment. Hematoxylin and eosin-stained sections were examined by two pathologists in a blind manner and a concordance was reached. The tumor differentiation was categorized as well-moderate or poor ( $>50$ vs. $\leq 50 \%$ gland formation). The tumor growth pattern at the tumor margin was defined as described previously (9).

Immunohistochemical analysis. Paraffin-embedded sections of NPC tissue samples were analyzed for the localization of NLK protein using an anti-NLK antibody (Rabbit anti-Human, 1:100 dilution; Sigma-Aldrich, St. Louis, MO, USA) as described previously (10). A tissue sample that was not treated with the primary antibody served as a negative control. The degree of immunohistochemical staining was evaluated independently by two pathologists blinded to the study and a consensus was reached. NLK nuclear immunoreactivity was scored using a semiquantitative scoring system: 0 , no staining; 1 , weak staining; 2 , moderate staining and 3 , strong staining. For statistical analysis, 0 and 1 were classified as NLK-negative; 2 and 3 were classified as NLK-positive.

Statistical analysis. Statistical analyses were performed with SPSS 16.0 (SPSS, Inc., Chicago, IL, USA). The $\chi^{2}$-square test was performed for categorical data. A Kaplan-Meier method and log-rank test were used for survival analyses. Univariate and multivariate Cox regression models were used to assess the correlations between the NLK status and the relative risks for relapse and mortality. The multivariate Cox regression models incorporated NLK expression and were adjusted according to the disease stage and histology. $\mathrm{P}<0.05$ was considered to indicate a statistically significant difference.
Table I. Clinicopathological characteristics of 352 patients with nasopharyngeal carcinoma.

\begin{tabular}{lc} 
Variables & Number of patients \\
\hline Gender (male/female, $\mathrm{n}$ ) & $204 / 148$ \\
T-primary tumor extent & 2 \\
T0 & 32 \\
T1 & 98 \\
T2 & 76 \\
T3 & 144 \\
T4 & \\
N-regional lymph nodes, $\mathrm{n}$ & 125 \\
N0-N1 & 227 \\
N2-N3 & \\
M-distant metastasis, $\mathrm{n}$ & 223 \\
M0 & 129 \\
M1 & \\
TNM stage, $\mathrm{n}$ & 101 \\
I-II & 251 \\
III-IV & \\
\hline
\end{tabular}

TNM, tumor, node and metastasis .

\section{Results}

Correlation of NLK with clinicopathological features. Patients included 204 males and 148 females, and the age at the time of diagnosis varied between 12 and 49 years (mean \pm standard deviation, $45.2 \pm 17.9$ years). The other clinicopathological characteristics of patients are shown in Table I.

NLK expression was classified as positive or negative by immunohistochemical analysis. Among all the 352 NPC biopsies, 54\% (190/352) of NPC samples were classified as positive and $46 \%(162 / 352)$ as negative for NLK expression. Representative images are shown in Fig. 1. Immunohistochemical staining of NLK was predominantly located in the nucleus. By contrast, NLK was negative in the adjacent tissue of all 176 specimens. The associations between the NLK status and other clinicopathological parameters are shown in Table II. The regional lymph node status was found to be significantly associated with the NLK status $(\mathrm{P}=0.003)$. Positive NLK expression was also correlated with distant metastases $(\mathrm{P}<0.001)$. Evident associations were not observed between the NLK status and tumor histology, or the gender of the patients.

NLK positivity predicted poor prognosis for the disease-free survival (DFS) in patients with NPC. With regard to DFS, among 286 patients with NPC for whom follow-up information was available, 134 patients (46.9\%) relapsed during the follow-up. In a Cox univariate regression analysis (Table III), a three-fold higher risk of recurrence was predicted for patients with NPC bearing tumors with NLK positivity [hazard ratio (HR), 2.94; 95\% confidence interval (CI), $1.30-6.52 ; \mathrm{P}=0.011]$. Therefore, in addition to the tumor 
Table II. Correlation between the NLK status and other clinicopathological variables.

\begin{tabular}{|c|c|c|c|c|}
\hline Variable & Total (n) & NLK-positive $(\mathrm{n}=190)$ & NLK-negative $(\mathrm{n}=162)$ & P-value \\
\hline Total patients & 352 & 190 & 162 & \\
\hline \multicolumn{5}{|l|}{ Gender } \\
\hline Male & 204 & 109 & 95 & \multirow[t]{2}{*}{0.79} \\
\hline Female & 148 & 81 & 67 & \\
\hline \multicolumn{5}{|l|}{ Histology } \\
\hline Undifferentiated & 185 & 114 & 71 & \multirow[t]{2}{*}{0.58} \\
\hline Non-keratinizing & 167 & 76 & 91 & \\
\hline \multicolumn{5}{|l|}{ Tumor extent } \\
\hline $\mathrm{T} 1-\mathrm{T} 2$ & 202 & 124 & 78 & \multirow[t]{2}{*}{0.016} \\
\hline T3-T4 & 150 & 66 & 84 & \\
\hline \multicolumn{5}{|c|}{ Regional lymph node status } \\
\hline N0-N1 & 114 & 79 & 35 & \multirow[t]{2}{*}{0.003} \\
\hline N2-N3 & 238 & 111 & 127 & \\
\hline \multicolumn{5}{|l|}{ Distant metastasis } \\
\hline M0 & 214 & 131 & 83 & \multirow[t]{2}{*}{$<0.001$} \\
\hline M1 & 138 & 59 & 79 & \\
\hline
\end{tabular}

NLK, nemo-like kinase.

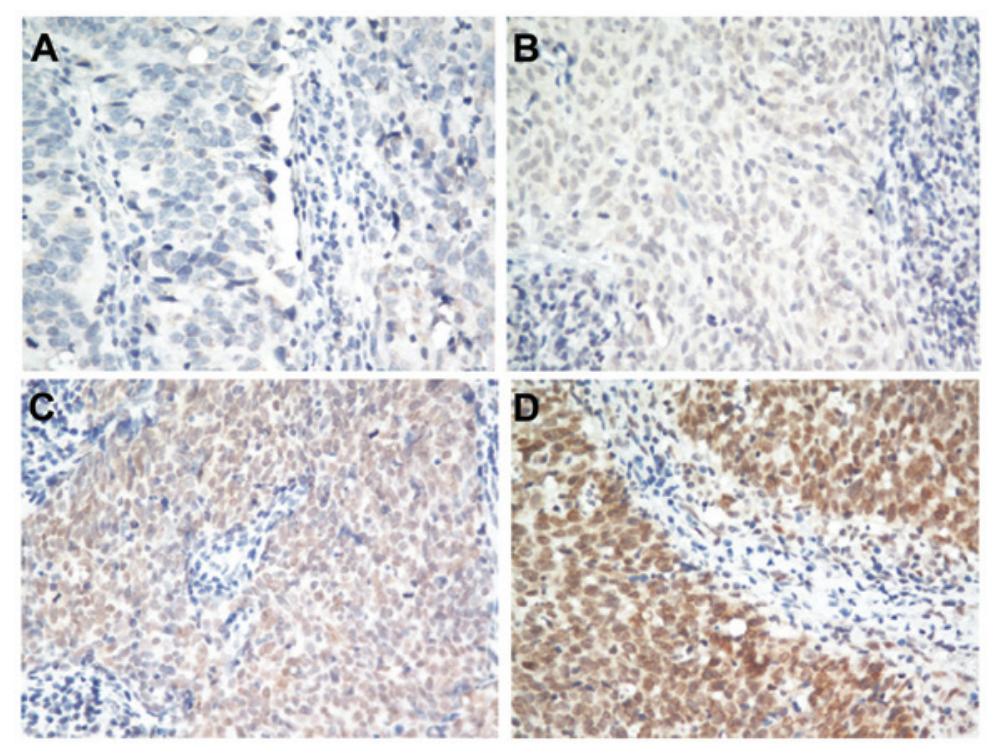

Figure 1. Immunohistochemical images of NLK staining patterns. NLK positivity was mainly localized within the nucleus. (A) Negative, (B) weak, (C) moderate and (D) strong staining [magnification, x400; staining, hemotoxylin (blue) and diaminobenzidine (brown)] in the nasopharyngeal carcinoma tissue. NLK, nemo-like kinase.

extent and TNM stage that were confirmed as significant predictors of DFS $(\mathrm{P}=0.034$ and $\mathrm{P}=0.014$, respectively), NLK overexpression was shown to predict shorter DFS in patients with NPC. In order to evaluate NLK positivity in terms of predicting the clinical outcome, the Kaplan-Meier survival analysis was also performed. In accordance, the Kaplan-Meier DFS curves demonstrated that patients with NPC with NLK-positive tumors had a significantly shorter DFS $(\mathrm{P}=0.0012)$ in comparison with the NLK-negative ones (Fig. 2A).
In the multivariate survival analysis (Table III), NLK positivity remained a statistically significant predictor of shorter DFS in patients with NPC, independent of other features of the patients, as patients with NLK-positive tumors were more prone to relapses (HR, 3.46; 95\% CI, 0.96-5.87; $\mathrm{P}=0.013$ ).

NLK positivity as an independent predictor of OS in patients with NPC. Among the 286 patients with NPC for whom follow-up data were available, 117 patients (40.9\%) succumbed to the disease during the follow-up. As demonstrated by Cox 
Table III. NLK positivity and survival of patients with nasopharyngeal carcinoma.

\begin{tabular}{|c|c|c|c|c|c|c|}
\hline \multirow[b]{2}{*}{ Variable } & \multicolumn{3}{|c|}{ Disease-free survival } & \multicolumn{3}{|c|}{ Overall survival } \\
\hline & HR & $95 \% \mathrm{CI}$ & P-value & HR & $95 \% \mathrm{CI}$ & P-value \\
\hline \multicolumn{7}{|c|}{ A, Univariate analysis } \\
\hline \multicolumn{7}{|l|}{ NLK } \\
\hline Negative & 1.00 & & & 1.00 & & \\
\hline Positive & 2.94 & $1.30-6.52$ & 0.001 & 2.77 & $1.12-5.98$ & 0.011 \\
\hline Gender & 1.02 & $0.56-2.09$ & 0.36 & 1.81 & $0.79-4.18$ & 0.16 \\
\hline Age & 1.21 & $0.61-2.73$ & 0.38 & 1.82 & $0.68-4.84$ & 0.23 \\
\hline Tumor histology & 0.79 & $0.28-1.45$ & 0.17 & 0.92 & $0.43-1.67$ & 0.43 \\
\hline Tumor extent & 1.96 & $1.01-3.93$ & 0.046 & 1.62 & $1.03-3.23$ & 0.034 \\
\hline Lymph node status & 1.21 & $0.94-1.98$ & 0.14 & 1.12 & $0.90-1.94$ & 0.26 \\
\hline TNM stage & 3.19 & $1.02-10.26$ & $<0.001$ & 1.31 & $1.31-5.48$ & 0.014 \\
\hline
\end{tabular}

B, Multivariate analysis

\begin{tabular}{|c|c|c|c|c|c|c|}
\hline \multicolumn{7}{|l|}{ NLK } \\
\hline Negative & 1.00 & & & 1.00 & & \\
\hline Positive & 3.46 & $0.96-5.87$ & 0.013 & 3.15 & $1.33-7.84$ & 0.019 \\
\hline Gender & 1.26 & $0.48-3.31$ & 0.64 & 1.66 & $0.55-4.99$ & 0.37 \\
\hline Age & 1.45 & $0.53-4.01$ & 0.46 & 1.88 & $0.59-5.95$ & 0.28 \\
\hline Tumor histology & 0.64 & $0.30-1.40$ & 0.28 & 0.61 & $0.26-1.40$ & 0.24 \\
\hline Tumor extent & 1.26 & $0.76-2.06$ & 0.37 & 1.34 & $0.79-2.29$ & 0.27 \\
\hline Lymph node status & 1.06 & $0.70-1.60$ & 0.78 & 1.07 & $0.68-1.70$ & 0.77 \\
\hline TNM stage & 2.70 & $1.68-4.94$ & $<0.001$ & 1.76 & $1.25-3.46$ & 0.027 \\
\hline
\end{tabular}

Age ( $\leq 30$ vs. $>30$ years), tumor histology (undifferentiated/non-keratinizing). Multivariate models were adjusted for gender, age, tumor histology, tumor extent, regional lymph node status and TNM stage. NLK, nemo-like kinase; HR, hazard ratio; CI, confidence interval; TNM, tumor, node and metastasis.

univariate regression analysis (Table III), patients with NPC with positive NLK were at higher risk of mortality $(\mathrm{HR}=2.77$, 95\% $\mathrm{CI}=1.12-5.98, \mathrm{P}=0.011$ ) compared with patients with NPC whose biopsies were NLK-negative. Consequently, enhanced NLK positivity was an unfavorable prognostic factor of OS. The tumor extent and TNM stage were also significant prognostic factors of $\mathrm{OS}(\mathrm{P}=0.034$ and 0.014 , respectively). In agreement with these results, the Kaplan-Meier OS curves demonstrated that patients with NPC with NLK-positive expression had a worse prognosis compared with patients with NLK-negative NPC ( $\mathrm{P}<0.001$; Fig. 2B).

In the multivariate Cox regression analysis (Table III), NLK positivity predicted a significantly unfavorable prognostic outcome (HR, 2.77; 95\% CI, 1.12-5.98; P=0.011). Of note, NLK positivity retained its independent prognostic significance in NPC (HR, 3.15; 95\% CI, 1.33-7.84; $\mathrm{P}=0.019)$, even when the multivariate Cox regression model was adjusted to the gender, age, tumor histology and TNM stage of patients.

Prognostic value of NLK positivity in patients with NPC without distant metastases. Since metastasis-free (M0) patients are substantially different from those with metastases in distant organs (M1), in terms of their prognosis and postoperative treatment, Kaplan-Meier survival analysis was conducted to evaluate the influence of NLK positivity on DFS and OS for metastasis-free patients with NPC. As shown in Fig. 3, M0 patients with NLK-positive tumors had a significantly shorter DFS and OS compared with M0 patients with NLK-negative malignancies $(\mathrm{P}<0.001$ and $\mathrm{P}=0.009$, respectively).

\section{Discussion}

To date, the prognosis of NPC has remained unsatisfactory and NPC represents an invasive and rapidly proliferating tumor $(3,4)$. Therefore, it is necessary to identify prognostic biomarkers that are independently correlated with tumor prognosis and aggressiveness. In the present study, the NLK expression status was examined by immunohistochemical analysis in 352 patients with NPC and compared with the survival rate and clinical and pathological features. The present study found NLK positivity in $~ 54 \%$ of patients. Additionally, significant correlations were identified between NLK positivity, poor differentiation and poor clinical outcome, independent of other characteristics. The results indicated that NLK positivity may be considered as a good prognostic marker for NPC. 
A

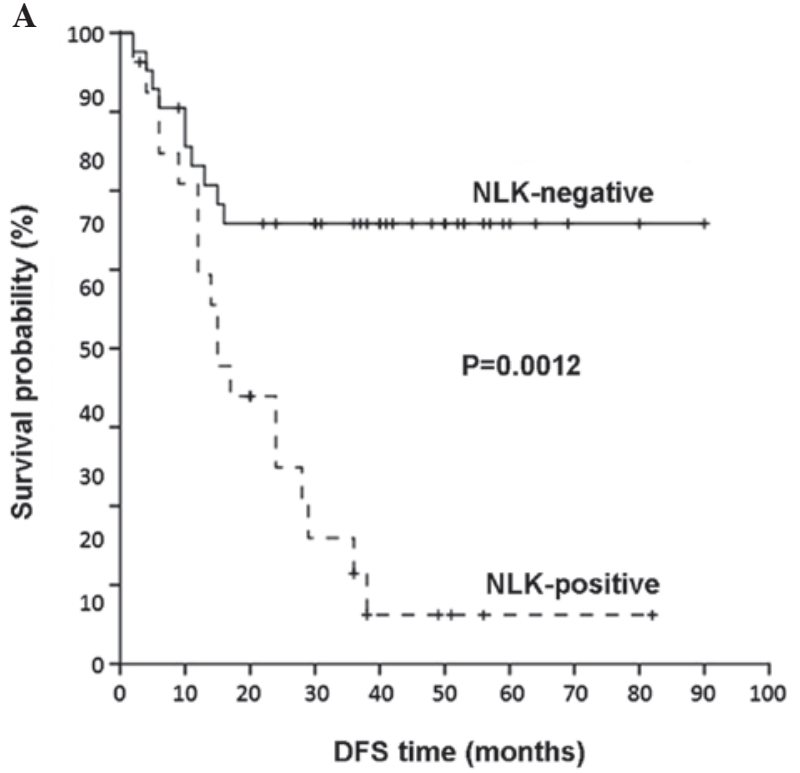

B

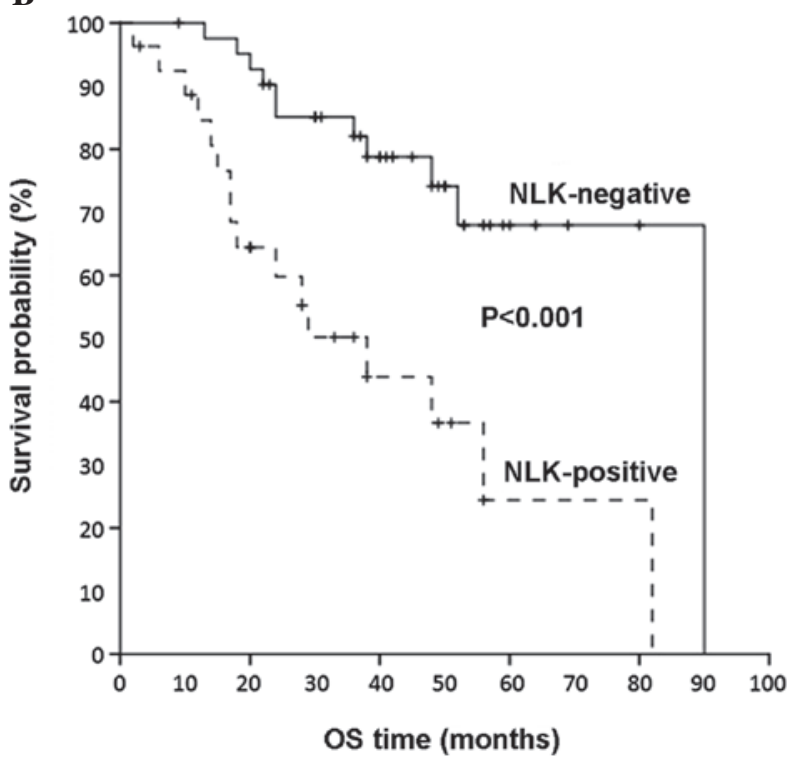

Figure 2. Kaplan-Meier curves for the overall survival according to NLK status in 352 patients with NPC stratified by nuclear NLK immunoreactivity (negative vs. positive). NLK positivity possesses an unfavorable prognostic value in NPC, as patients with NLK-positive nasopharyngeal tumors have significantly shorter (A) DFS and (B) OS, compared with NPC patients with NLK-negative tumors. NPC, nasopharyngeal carcinoma; NLK, nemo-like kinase; DFS, disease-free survival; OS, overall survival.

The functions of NLK in human tumors remain to be fully elucidated and are subject to current studies. In previous studies, NLK has been shown to function as a tumor suppressor gene (11-13). For example, the overexpression of NLK in the DLD-1 human colon cancer cell line inhibited cell proliferation and increased the number of apoptotic cells (11). Subsequent studies found that NLK expression was decreased during prostate cancer metastases and demonstrated that overexpression of NLK resulted in induction of apoptosis (12). In a clinical analysis examining specimens from 70 human gliomas by immunohistochemical and western blot analysis, a low NLK expression level was associated with poor patient outcome (13). However, other studies

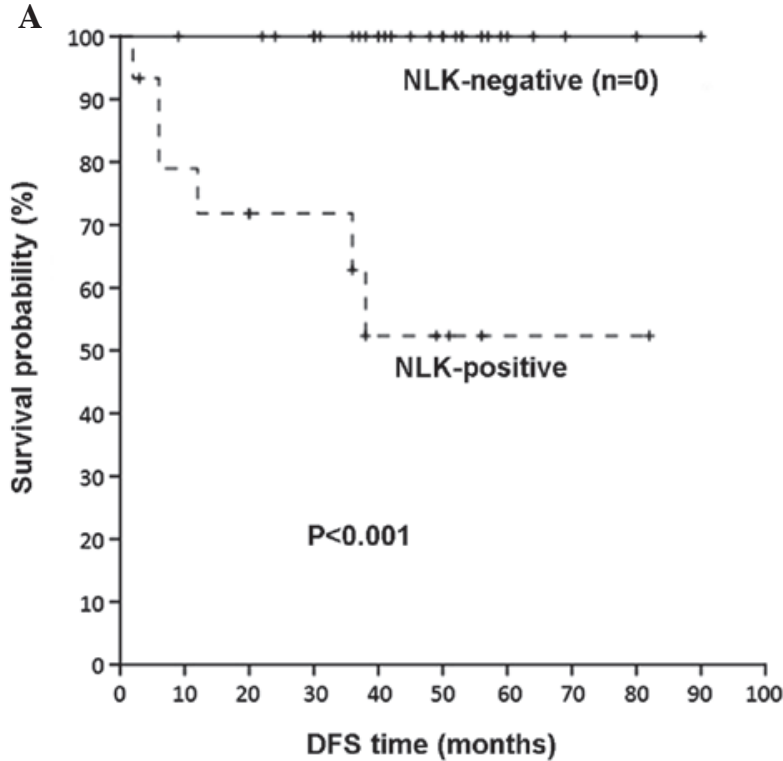

B

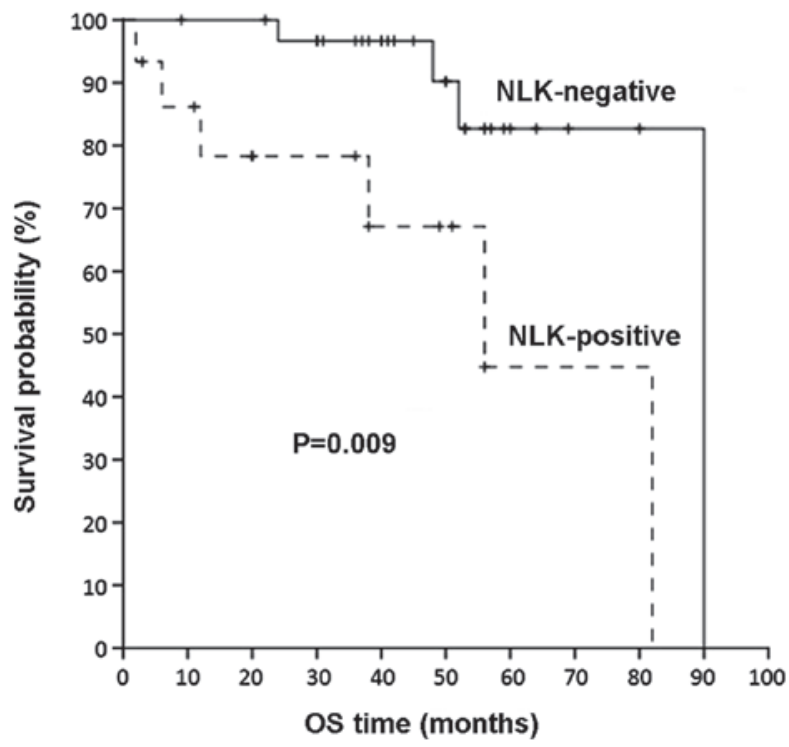

Figure 3. Kaplan-Meier survival curves for patients with NPC without distant metastases. NLK-positive patients without metastatic nasopharyngeal tumors have a significantly lower (A) DFS and (B) OS in comparison with metastasis-free patients bearing NLK-negative tumors. NPC, nasopharyngeal carcinoma; NLK, nemo-like kinase; DFS, disease-free survival; OS, overall survival.

reported converse results which showed that NLK acts as an oncogene in a number of tumors (6-8). NLK has a mitogenic potential in hepatocellular carcinomas, as siRNA-mediated disruption of NLK inhibited the proliferation of Hep3B cells and arrested cell cycle transition (6). NLK silencing inhibited the growth rate and alleviated the migration ability of gallbladder carcinoma cell lines (7). A subsequent clinical investigation showed that the overexpression of NLK is an independent prognostic factor for patients with gallbladder carcinoma (8). The mechanisms underlying all these discrepancies remain to be elucidated.

In the present study, a relatively large cohort of samples from patients with NPC $(n=352)$ was investigated. NLK positivity was observed in $\sim 54 \%$ of patients with NPC, while normal controls were negatively stained for NLK. Furthermore, 
Cox regression analysis revealed that NLK positivity was an unfavorable prognostic indicator of DFS and OS in patients with NPC, independent of other features. Additionally, NLK-positive patients with NPC without distant metastases are more likely to relapse compared with NLK-negative patients with NPC without distant metastases.

In conclusion, NLK is an independent prognostic factor for the survival of patients with NPC and NLK may be a potential prognostic biomarker for clinical applications in patients with NPC. Clinical prospective studies of NLK as a novel biomarker in NPC are warranted.

\section{References}

1. Wei KR, Xu Y, Liu J, Zhang WJ and Liang ZH: Histopathological classification of nasopharyngeal carcinoma. Asian Pac J Cancer Prev 12: 1141-1147, 2011.

2. Zhen Y, Ye Y, Yu X, Mai C, Zhou Y, Chen Y, Yang H, Lyu X, Song Y, Wu Q, Fu Q, Zhao M, Hua S, Wang H, Liu Z, Zhang Y and Fang W: Reduced CTGF expression promotes cell growth, migration, and invasion in nasopharyngeal carcinoma. PLoS One 8: e64976, 2013.

3. Ho FC, Tham IW, Earnest A, Lee KM and Lu JJ: Patterns of regional lymph node metastasis of nasopharyngeal carcinoma: a meta-analysis of clinical evidence. BMC Cancer 12: 98, 2012.

4. Jia WH and Qin HD: Non-viral environmental risk factors for nasopharyngeal carcinoma: a systematic review. Semin Cancer Biol 22: 117-126, 2012.

5. Brott BK, Pinsky BA and Erikson RL: NLK is a murine protein kinase related to Erk/MAP kinases and localized in the nucleus. Proc Natl Acad Sci USA 95: 963-968, 1998.
6. Jung KH, Kim JK, Noh JH, Eun JW, Bae HJ, Xie HJ, Ahn YM, Park WS, Lee JY and Nam SW: Targeted disruption of Nemo-like kinase inhibits tumor cell growth by simultaneous suppression of cyclin D1 and CDK2 in human hepatocellular carcinoma. J Cell Biochem 110: 687-696, 2010.

7. Tan Z, Li M, Wu W, Zhang L, Ding Q, Wu X, Mu J and Liu Y: NLK is a key regulator of proliferation and migration in gallbladder carcinoma cells. Mol Cell Biochem 369: 27-33, 2012.

8. Li M, Zhang S, Wang Z, Zhang B, Wu X, Weng H, Ding Q, Tan Z, Zhang N, Mu J, Yang J, Shu Y, Bao R, Ding Q, Wu W, Cao Y and Liu Y: Prognostic significance of nemo-like kinase (NLK) expression in patients with gallbladder cancer. Tumour Biol: Jul 16, 2013 (Epub ahead of print).

9. Morikawa T, Kuchiba A, Qian ZR, Mino-Kenudson M, Hornick JL, Yamauchi M, Imamura Y, Liao X, Nishihara R, Meyerhardt JA, Fuchs CS and Ogino S: Prognostic significance and molecular associations of tumor growth pattern in colorectal cancer. Ann Surg Oncol 19: 1944-1953, 2012.

10. Morikawa T, Kuchiba A, Yamauchi M, Meyerhardt JA, Shima K, Nosho K, Chan AT, Giovannucci E, Fuchs CS and Ogino S: Association of CTNNB1 (beta-catenin) alterations, body mass index, and physical activity with survival in patients with colorectal cancer. JAMA 305: 1685-1694, 2011

11. Yasuda J, Tsuchiya A, Yamada T, Sakamoto M, Sekiya T and Hirohashi S: Nemo-like kinase induces apoptosis in DLD-1 human colon cancer cells. Biochem Biophys Res Commun 308: 227-233, 2003.

12. Emami KH, Brown LG, Pitts TE, Sun X, Vessella RL and Corey E: Nemo-like kinase induces apoptosis and inhibits androgen receptor signaling in prostate cancer cells. Prostate 69: 1481-1492, 2009

13. Cui G, Li Z, Shao B, Zhao L, Zhou Y, Lu T, Wang J, Shi X, Wang J, Zuo G, Zhu W and Shen A: Clinical and biological significance of nemo-like kinase expression in glioma. J Clin Neurosci 18: 271-275, 2011 